\title{
Numerical Modelling of Axially Loaded Helical Piles: Compressive Resistance
}

\author{
Ahmed. S. Ali ${ }^{1, a}$, Nahla. M. Salim ${ }^{1, b^{*}}$, and Husam. H. Baqir ${ }^{1, \mathrm{c}}$ \\ ${ }^{1}$ Civil Engineering Department, University of Technology, Baghdad, Iraq \\ abce.19.61@grad.uotechnology.edu.iq, ${ }^{\mathrm{b}} 40068 @$ uotechnology.edu.iq, ${ }^{\mathrm{c}} 40161 @$ uotechnology.edu.iq
}

\begin{abstract}
Helical piles are foundation systems used to support compression, tension, and lateral loads. However, this type of piles was used around the world for more than 25 years. Its behavior, especially in Iraq, is still unknown and scare. The present study is carried out by analyses of this type of pile using the finite element method. Modeling of the helical pile geometry has been proposed using the finite element through the computer program Plaxis 3D. Parametric analyses were also performed. The main parametric study is the effect of a number of the helix, spacing between helix, the helix diameter, and helix configuration. The main conclusion is that as the number of helix increases, the bearing capacity increases further more than the higher the distance between helix, the higher bearing capacity. Maximum pile capacity with the case of three-helix increased by 115.4 $\%$ compared to the case without helix. Pile capacity with the case of spacing $3.5 \mathrm{D}$ reached $130.7 \%$ compared to the case of spacing $0.5 \mathrm{D}$. The value of displacement decreased with increasing spacing between the helices, while the value of displacement increased with the decrease in the spacing between the helices for top, middle, and bottom helix.
\end{abstract}

Keywords: Helical pile, Numerical model, Number of a helix ,Helix spacing, Changing diameter helix, Effect location of a helix.

\section{Introduction}

Helical piles are one of the types of deep piles that are used to support foundations that are located in a load of marine as tension and compression member, as well as used as a lateral tension member to support retaining walls and earthen embankments to preserve them from collapses that occur. A helical pile is shaped to have helical plates with a diameter greater than the shaft attached to the hollow shaft and respective helix plates with end bearing capacity and shaft frictional capacities to achieve the bearing capacity [1]. The helical pile is calculated in cylindrical or individual mode to know the bearing capacity of the helical pile. As cylindrical, all helices work together to find the bearing capacity. Every helix works separately as an individual mode, and the end bearing equals the sum of each helical end bearing. The Irish civil engineer Alexander Mitchell invented helical piles in 1836 to strengthen housing foundations. Since 1853, helical piles have served in the UK and generally served as a basis for lightweight houses in the US between 1850 and 1890 . Helical piles were then used for anchor-like purposes until 1985[1]. The conduct of the helical pile was studied by AbdelRahim et al. [2]. With the increase in the helix diameter, they found the compression loads to increase [2].

Sprince and Pakrastince [3] concluded that the helical pile capacity affects the diameter of the capacitive plate, although it is not the same in every soil. The diameter of the helix has been obtained by Elsherbiny and El Naggar [4] 2-3 times that of the shaft diameter. Therefore, a helical pile with one helix would have 4 to 9 times the end-bearing resistance of a conventional pile for the same pile embedding, shaft diameter, and soil strength parameters. The effect of the helical pile behavior in the sand was studied by Salhi et al. [5]. Salhi et al. [5] concluded that the load transfer from the low helix to the middle and top helix is being achieved through an increasing spacing ratio. The capacity increases considerably as the spacing ratio increases. The first study parameter is that the helix spacing influences the pile capacity, depending upon the ratio of spacing to helix diameter (S/D) [6]. Khalaf [6] concluded that multi helices (triple helices) screw piles provided greater capacity than single and double helices, so the uplift and compression resistance of the pile is kept increasing with the increasing number of helices. El Naggar and Elkasabgy [7] found that soil disturbance had a major effect on the mechanism of pile failure during pile installation, regardless of the inter-helix spacing 
to helix diameter value. Sisikar [8] found the transition from cylindrical shear to individual plates with a value of the spacing ratio through a study of the load transfer mechanism (1.5 to 2). The main conclusion is drawn that the load transition from cylindrical shear to individual plate occurs at a spacing ratio range from 1.5 to 2.0 .

The objectives of this paper are to Simulate the behavior of helical piles using 3D Plaxis software (Plaxis 2020), the bearing capacity of the pile with helix and without helix, and the effect of spacing between helices the distance of helix and helices configuration.

\section{Verification of Program (PLAXIS 3D 2020)}

A comparison was made between an experimental work [9] and simulated through a numerical model. Figure 1 shows the load-displacement $(\mathrm{Uz})$ for the numerical model and experimental work. According to this figure, there is convergence in shape and results of values between the numerical model and experimental work. By using a tangent method that illustrated by Butler and Hoy [9], the maximum displacement $(\mathrm{Uz})$ and maximum load in the numerical model are $-0.8 \mathrm{~mm}$ and $0.61 \mathrm{kN}$, respectively, while maximum displacement (Uz) and maximum load in the experimental work are $0.8 \mathrm{~mm}$ and $0.72 \mathrm{kN}$ respectively. By comparing the results, it was noticed that there are very similar results in the displacement value, and this is evidence that the program gives excellent results that simulate the experimental work.

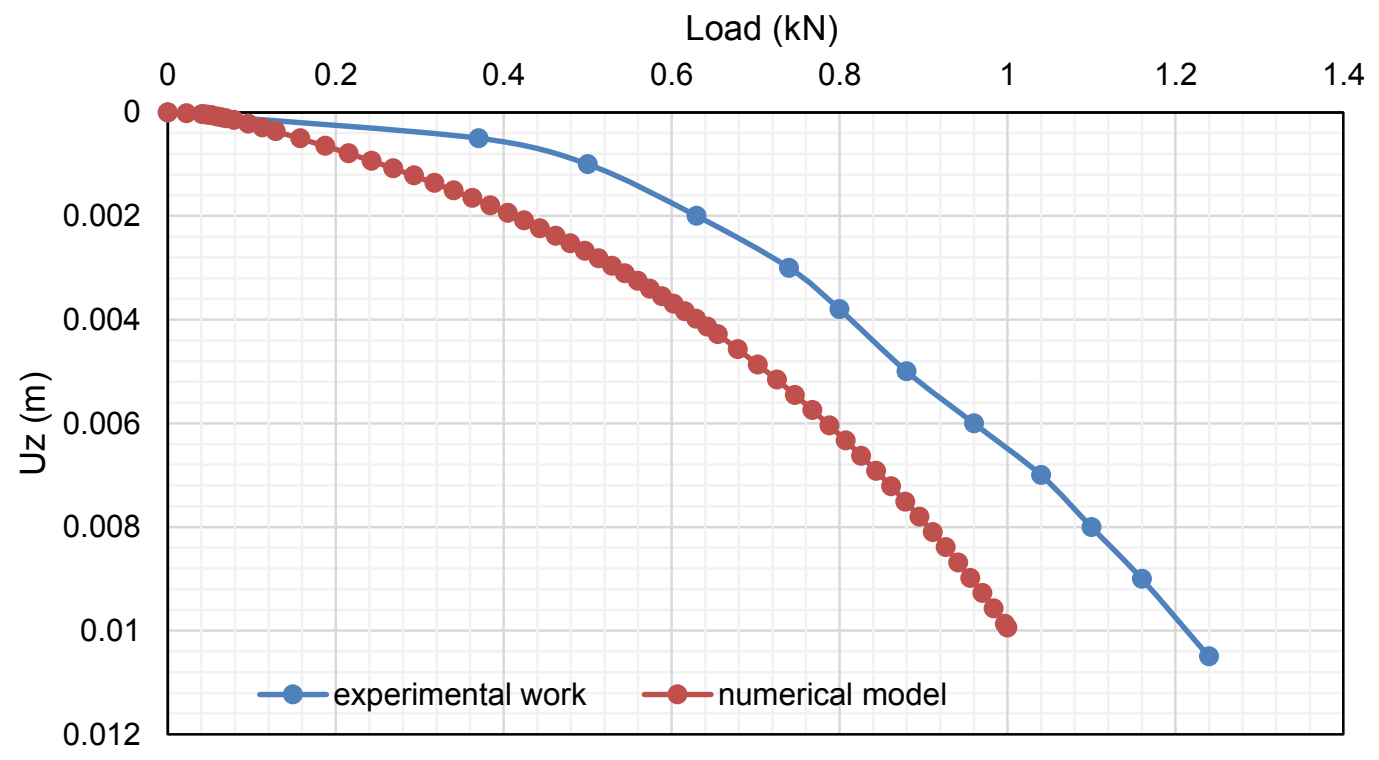

Figure 1. Simulation of experimental work with a numerical model for a helical pile of one helix under compression loading in saturated soil.

\section{Methodology and Preparation Model}

In this paper, a 3D finite element program (PLAXIS) is used. The structural properties of the model for this parametric study are used. The diameter of a cup of helical pile is $0.325 \mathrm{~m}$. The length of the helical pile is $10 \mathrm{~m}$. the diameter of the shaft and thickness are $0.325 \mathrm{~m}, 9.5 \mathrm{~mm}$, respectively. The diameter of the helix and its thicknesses are $0.762 \mathrm{~m}$ and $25 \mathrm{~mm}$ respectively. A maximum of three helices was used. Medium sand of soil with dimensions $10 \times 10 \mathrm{~m}^{2}, 35 \mathrm{~m}$ depth, and $\mathrm{RD}=50 \%$. The static load is applied on the helical pile according to Eq. (1) [9].

$$
\mathrm{Q}_{\mathrm{C}}=1 / 2 \pi \mathrm{Da} \gamma\left(\mathrm{H}_{\mathrm{b}}^{2}-\mathrm{H}_{\mathrm{t}}^{2}\right) \mathrm{Ks} \tan \varphi+\gamma \mathrm{H} \mathrm{A}_{\mathrm{h}} \mathrm{Nq}+1 / 2 \mathrm{Ps} \mathrm{H}_{\mathrm{eff}^{2}} \gamma \mathrm{Ks} \tan \varphi
$$

Where:

$\mathrm{Qc}=$ Ultimate compression load $(\mathrm{kN})$.

Qhelix $=$ Bearing strength of helix.

$Q_{\text {bearing }}=$ Bearing strength of the base. 
$\mathrm{Q}_{\text {shaft }}=$ Strength of shaft only.

$\mathrm{Da}=$ Average helix diameter.

$\mathrm{H}_{\mathrm{b}}=$ Depth bottom helix.

$\mathrm{H}_{\mathrm{t}}=$ depth to top helix.

$\mathrm{H}=$ Embedment depth of pile.

$\mathrm{D}=$ Diameter of pile helix.

$\mathrm{H}_{\text {eff }}=$ Effective length of pile above top helix $\left(\mathrm{H}_{\mathrm{eff}}=\mathrm{H}-\mathrm{D}\right)$.

$\mathrm{d}=$ diameter of shaft.

$\mathrm{A}_{\mathrm{h}}=$ Area of a helix $\left(\mathrm{m}^{2}\right)$.

$\mathrm{Ks}=$ Coefficient of lateral earth pressure in compression loading [9].

$\mathrm{Ps}=$ Perimeter of the helical pile shaft.

$\varphi=$ The angle of internal friction.

$\mathrm{Nq}=$ Bearing capacity factor of soil [9].

Figure 2 shows the dimensions of the helical pile and its details. Figure 3. indicates the helical pile models in different configurations. PLAXIS 3D is a three-dimensional program for deformation, stability, and flow analyses for different types of geotechnical applications. The program uses a comfortable graphical user interface to quickly create a geometry model and a finite element mesh [10]. The soil parameters are listed in Table 1. Shafts and helices are modeled as plate materials. Two constitutive models were used to represent the materials. The "elastic" was assumed for the piles and helices, as shown in figure 4. The sand was represented by the hardening soil model. Results from laboratory tests were used to define the parameters needed for the numerical model [9]. A vertical static load is applied to the helical pile as a point load. Figure 4 depicts the numerical model, which includes the helical pile embedded in the sand.

After that, the same soil was used, but using the Mohr- Coulomb model, with the use of Young's Modulus (E) $30290 \mathrm{kN} / \mathrm{m}^{2}$ and Poisson's ratio (v) 0.3 for medium sand soils with top, middle, and bottom helix for three helices.

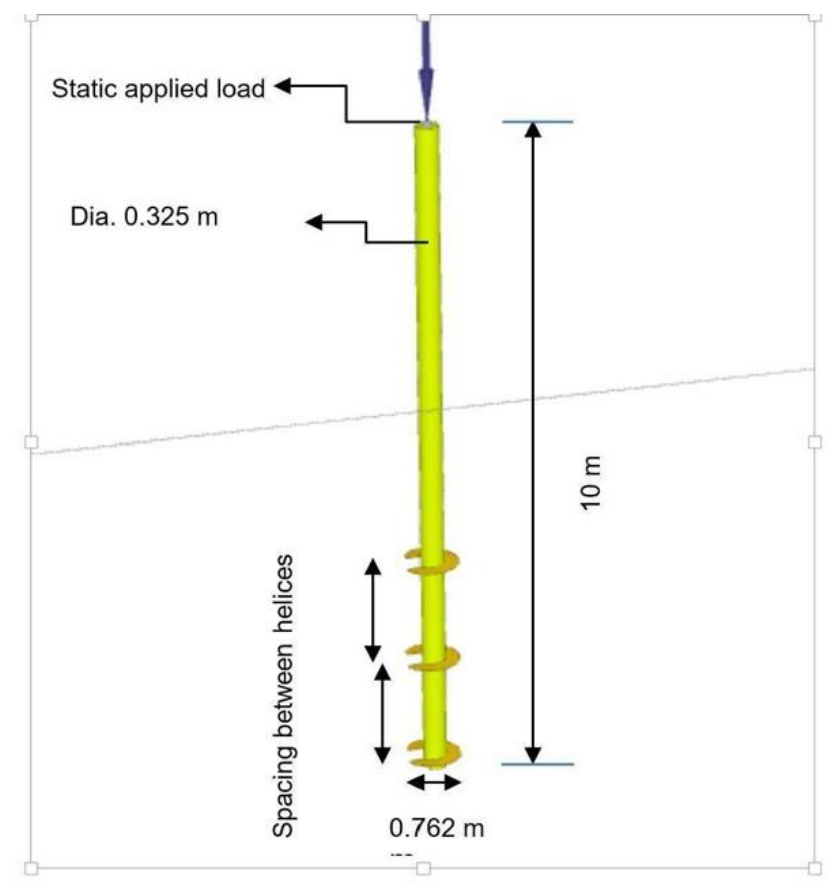

Figure 2. The dimensions of the helical pile and its details. 


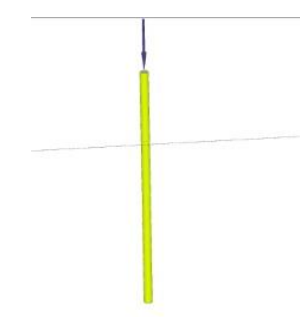

a) without helix

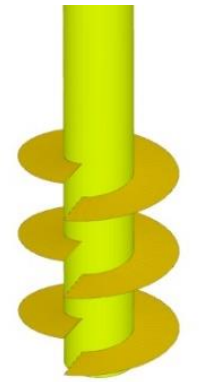

d) three helices $\mathrm{s}=0.5 \mathrm{~d}$

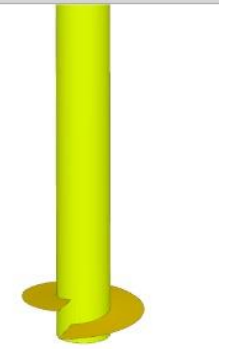

b) one helix

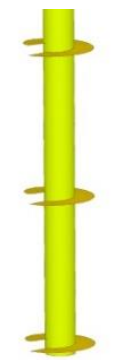

e) three helices $\mathrm{s}=3.5 \mathrm{~d}$

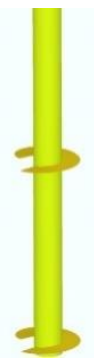

c) two helices $\mathrm{s}=3.5 \mathrm{~d}$

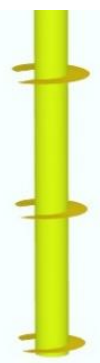

f) three helices with different diameter helix

Figure 3. Details of the helical pile.

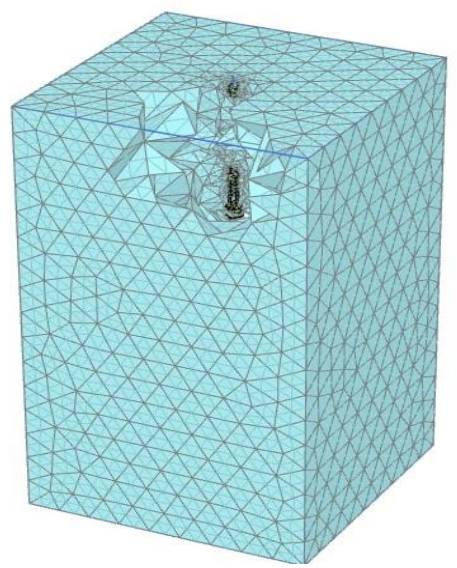

Figure 4. Mesh of numerical model soil with the helical pile.

Table 1. The parameters of the soil.

\begin{tabular}{cc}
\hline Properties & Medium Sand \\
\hline Model & Hardening soil \\
Unit weight, $\gamma\left(\mathrm{kN} / \mathrm{m}^{3}\right)$ & 19.77 \\
E50 ref. & $12.12 \mathrm{E} 3$ \\
Eoed ref. & $21.34 \mathrm{e} 3$ \\
Eur $_{\text {ref. }}$ & $36.35 \mathrm{e} 3$ \\
Angle of friction, $\varphi$ & 25 \\
Dilatancy angle, $\psi$ & 0 \\
R $_{\text {inter }}$ & 0.7 \\
\hline
\end{tabular}

\section{Result and Discussion}

According to reference, table 2 shows the maximum pile capacity without helix, one helix, two helices, and three-helix [9]. However, the main criterion used to determine the ultimate pile capacity is a slope tangent method with a crossing point of a line drawn tangent to the primary linear portion curve and the steeper portion curve of the load-settlement curve. This intersection represents the value of the failure load and explains how the non-linear part of the load-settlement curve has been settled [9]. 
Table 2. The maximum pile capacity is without helix, one helix, two helices, and three-helix.

\begin{tabular}{cccccccc}
\hline Model & \multicolumn{7}{c}{ Maximum pile capacity $\left(\mathbf{k N} / \mathbf{m}^{\mathbf{2}}\right)$} \\
\cline { 2 - 8 } & $0.5 \mathrm{D}$ & $1 \mathrm{D}$ & $1.5 \mathrm{D}$ & 2D & $2.5 \mathrm{D}$ & $3 \mathrm{D}$ & $3.5 \mathrm{D}$ \\
\hline Without helix & 1300 & 1300 & 1300 & 1300 & 1300 & 1300 & 1300 \\
One helix & 1325 & 1325 & 1325 & 1325 & 1325 & 1325 & 1325 \\
Two helix & 1200 & 1275 & 1300 & 1300 & 1375 & 1425 & 1500 \\
Three helix & 1300 & 1375 & 1425 & 1450 & 1615 & 1650 & 1700 \\
\hline
\end{tabular}

The effect number of helix. Figure 5 shows the compressive stress- displacement (with z-direction) for different cases (without helix, one helix, two helices, and three-helix). According to figure 5, maximum pile capacity in one helix increased by $102 \%$, but in two helices with $\mathrm{S}=2 \mathrm{D}$ increased by $107.7 \%$, while in the case of three-helix maximum pile capacity increased by $115.4 \%$. All these mention cases were compared to the case of the pile without helix.

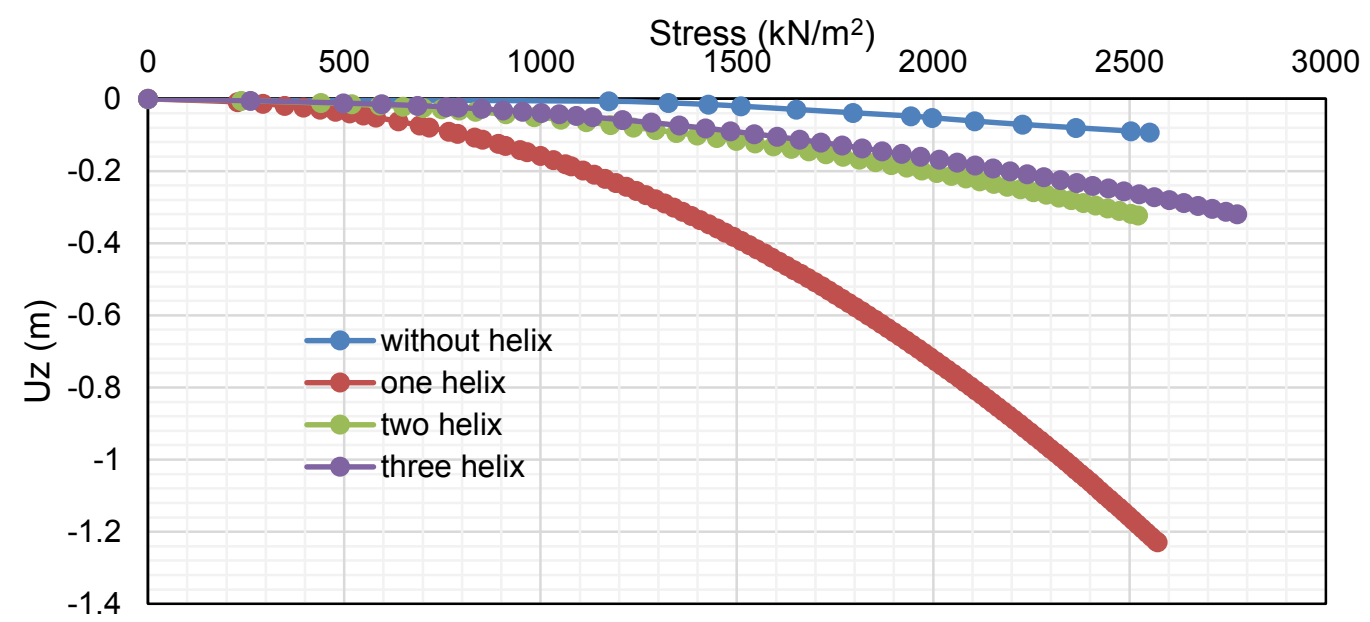

Figure 5. Stress-displacement under compression static load without helix, one helix, two helices, and three helices.

The effect spacing between helices. Figure 6 describes a comparison for different spacing between helixes, using two helices with spacing corresponding to $0.5 \mathrm{D}, 1 \mathrm{D}, 1.5 \mathrm{D}, 2 \mathrm{D}, 2.5 \mathrm{D}, 3 \mathrm{D}$, and 3.5D. According to this figure, the value of pile capacity with spacing 1D increased by $106.25 \%$ than the value of pile capacity with spacing $0.5 \mathrm{D}$, while the percentage in case $1.5 \mathrm{D}$ increased by $108.3 \%$ than from case of $0.5 \mathrm{D}$, until the percentage reached $125 \%$ with spacing $3.5 \mathrm{D}$. Figure 7 describes a comparison for different spacing between helical piles with three helices. As a result, the pile capacity of three helices with spacing $1 \mathrm{D}$ increased by $105.7 \%$ compared to the case of $0.5 \mathrm{D}$, while pile capacity with $3.5 \mathrm{D}$ reached $130.7 \%$.

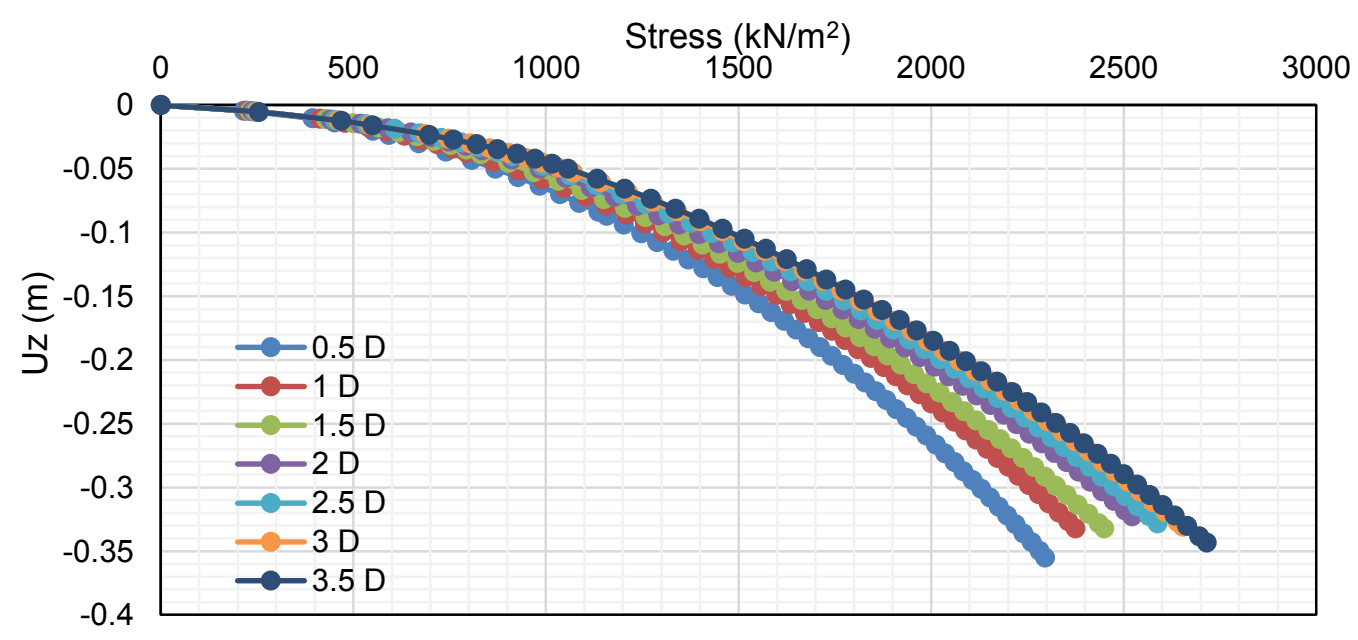

Figure 6. Stress-displacement under compression oad with two helices for different spacing. 


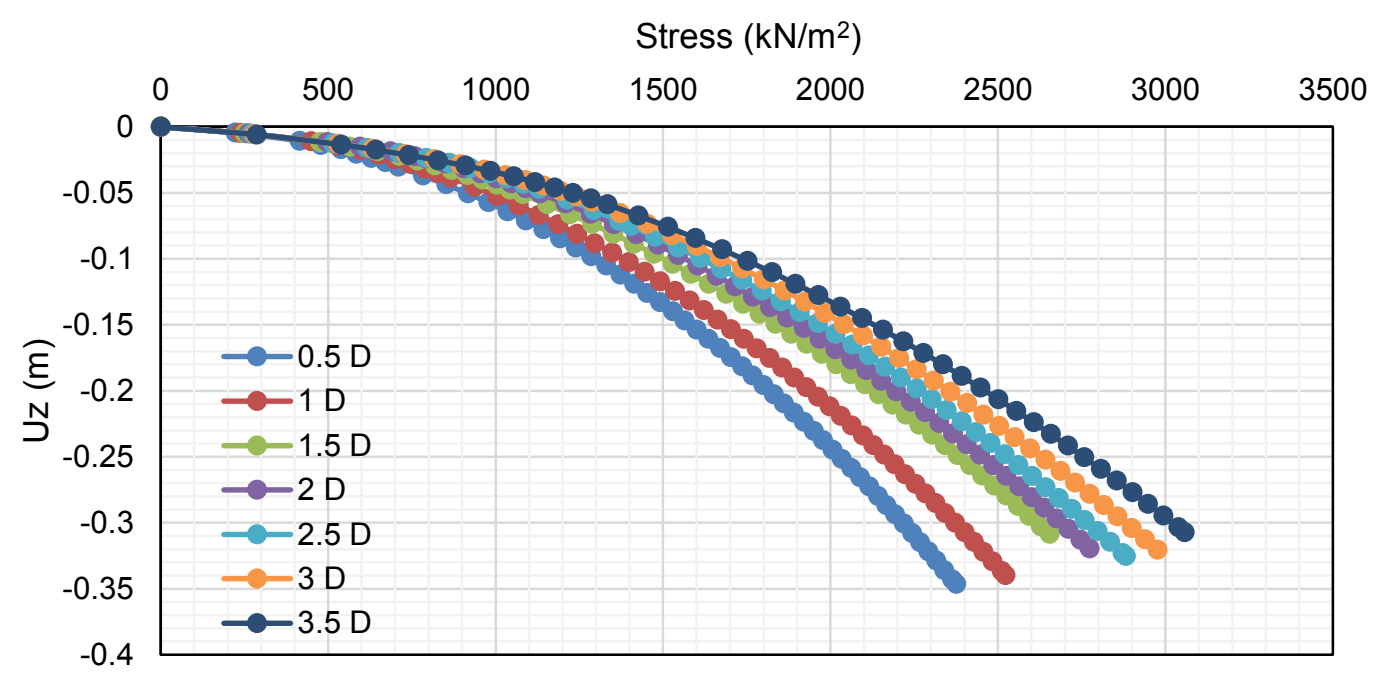

Figure 7. Stress-displacement under compression static load with three helices for different spacing.

Figure 8 shows the shape of deformation of displacement with direction z-axis. According to this figure, the shape of the deformation is concentrated around the helical pile, especially around the helices, because the helices and shaft pile cause deformation in the soil in contact with them under compression load, and this deformation is minor does not cause failure in the soil.

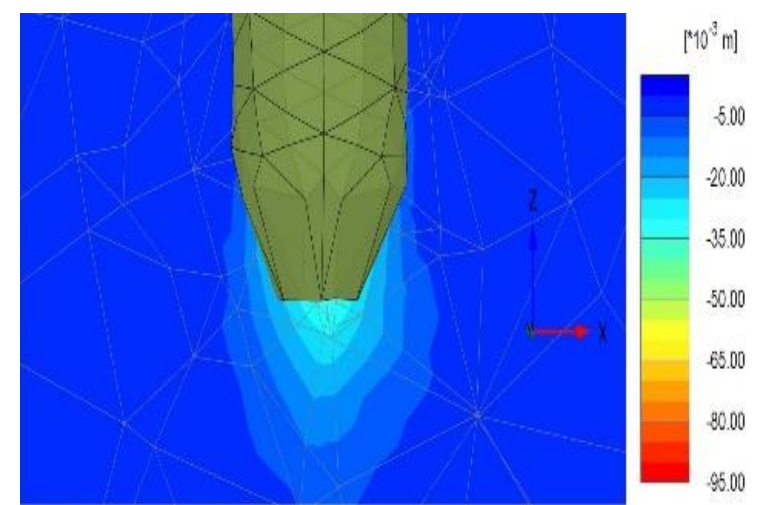

a) Without helix

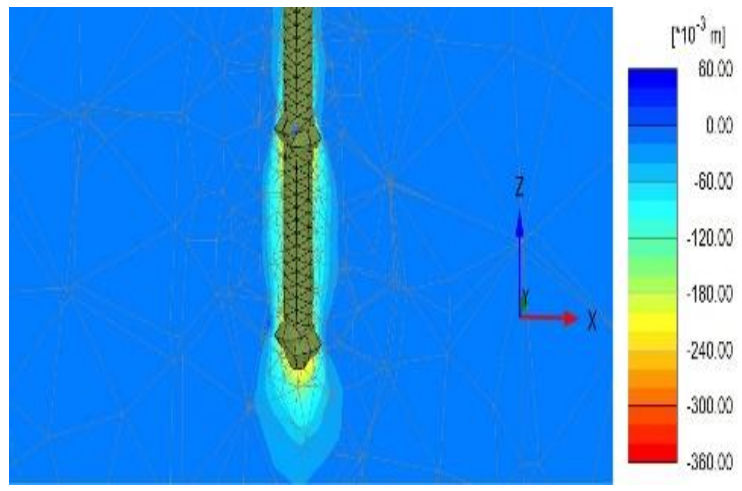

c) Two helices $\mathrm{S}=3.5 \mathrm{D}$

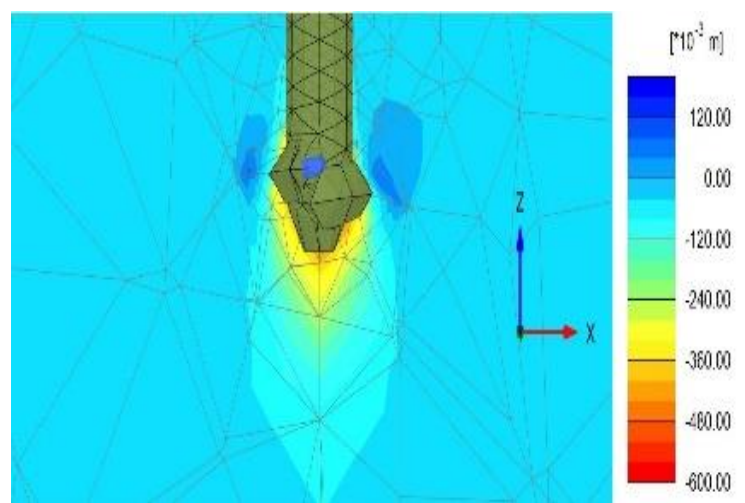

b) One helix

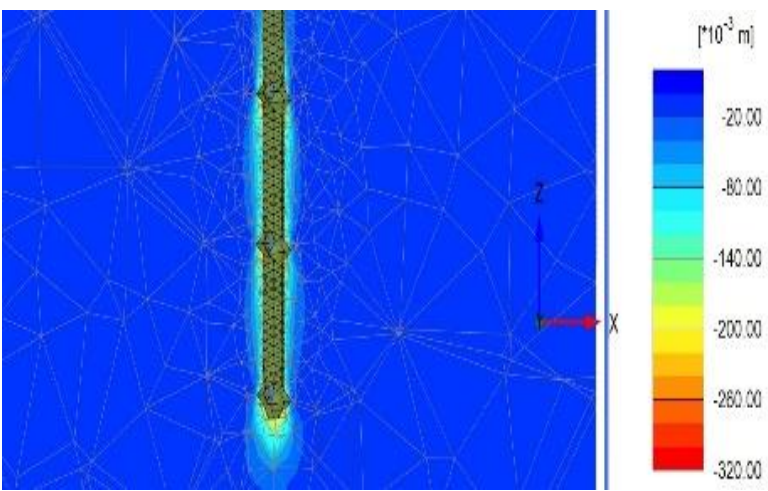

d) Three helices $\mathrm{S}=3.5 \mathrm{D}$

Figure 8. Deformation shapes of modeled helical piles.

Effect changing the diameter of helices. The change in diameter of helices was simulated in two ways:

1) Pile with uniformly sized plates was simulated, and the ultimate capacity is shown in Figure 9a. 
2) Pile with tapered plates (diameter decreasing with increasing depth were simulated, and the results are shown in Figures 9-b.

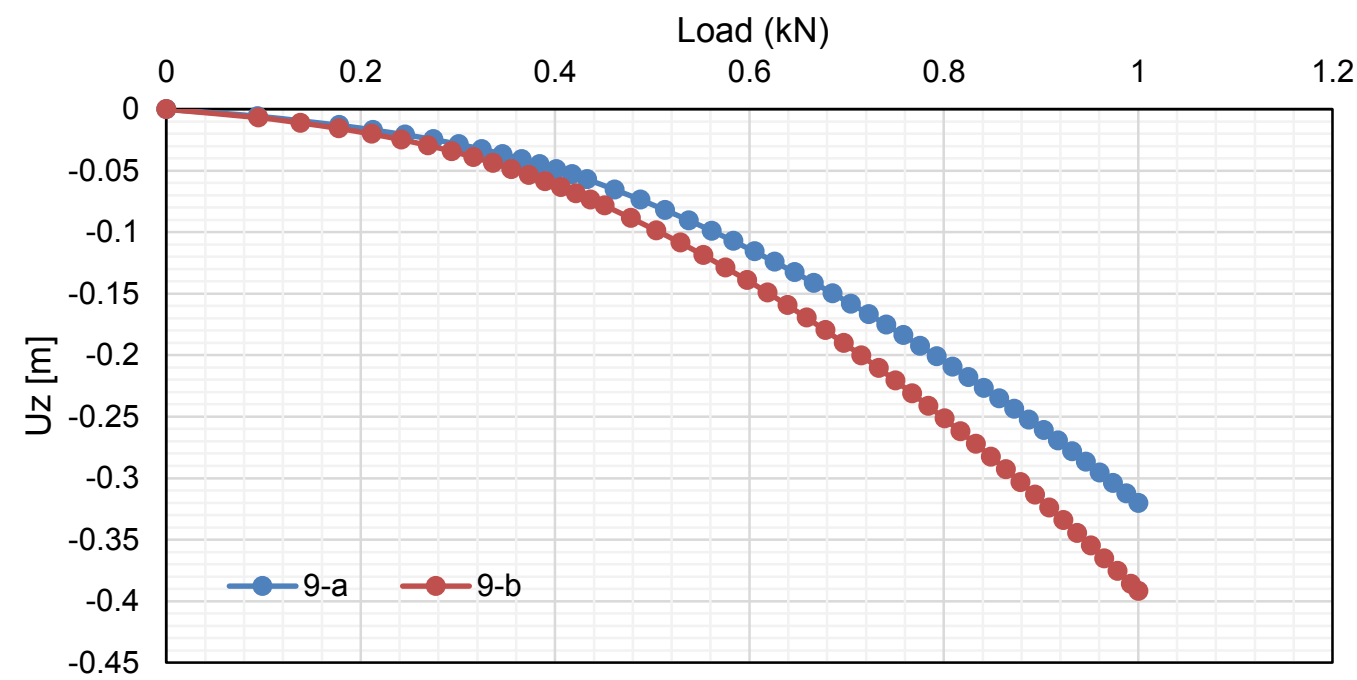

Figure 9. Load-displacement under compression static load with three helices for a) with the same diameter of the helix and b) with different helix diameters.

Effect of the location of the helix on the behavior of soil. Figure 10 shows the effect of the helix location on the displacement of the soil. According to this figure, the value of displacement in the case of the top helix is more than in the case of a middle and bottom helix.

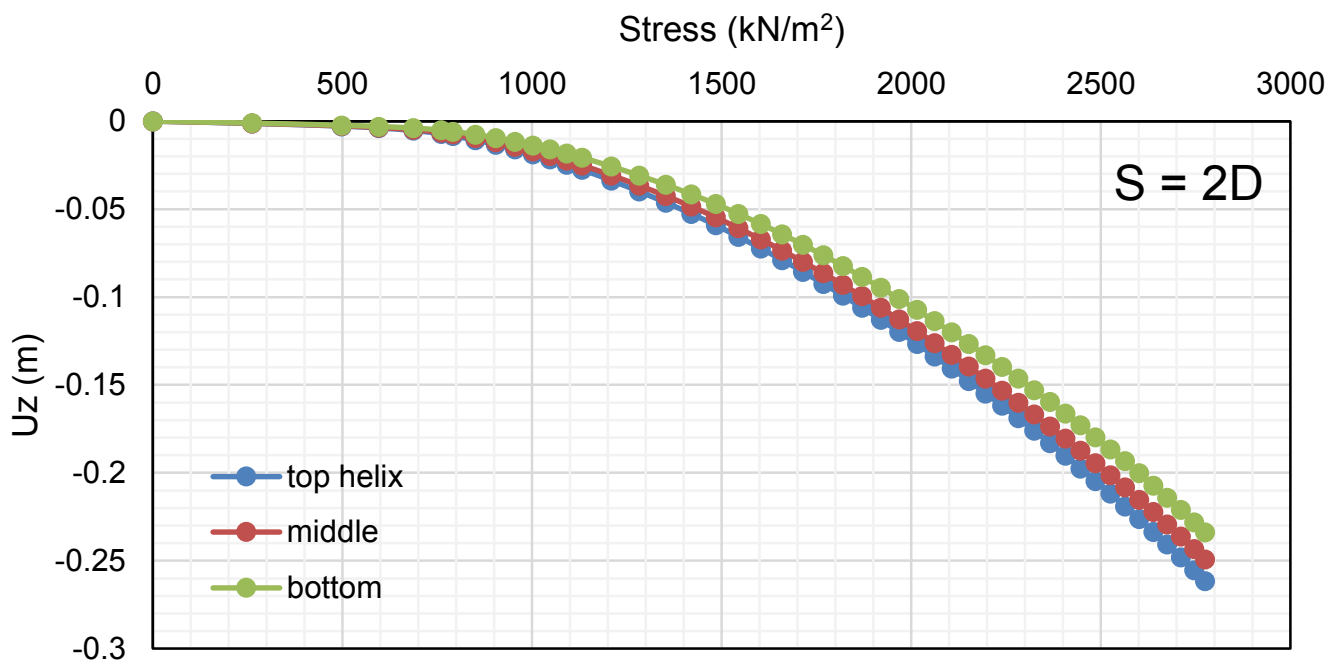

Figure 10. Stress-displacement under compression static load with three helices for a) top helix, b) middle helix, c) bottom helix.

Using the Mohr-Coulomb model to study the effect of the location of the helix. Figure 11 describes the effect of the helix location on the load-displacement of the soil with different spacing between helices. According to this figure, the value of displacement in the case of the 3.5D is more than in the case of $0.5 \mathrm{D}$ in all cases (top, middle, and bottom helix). 

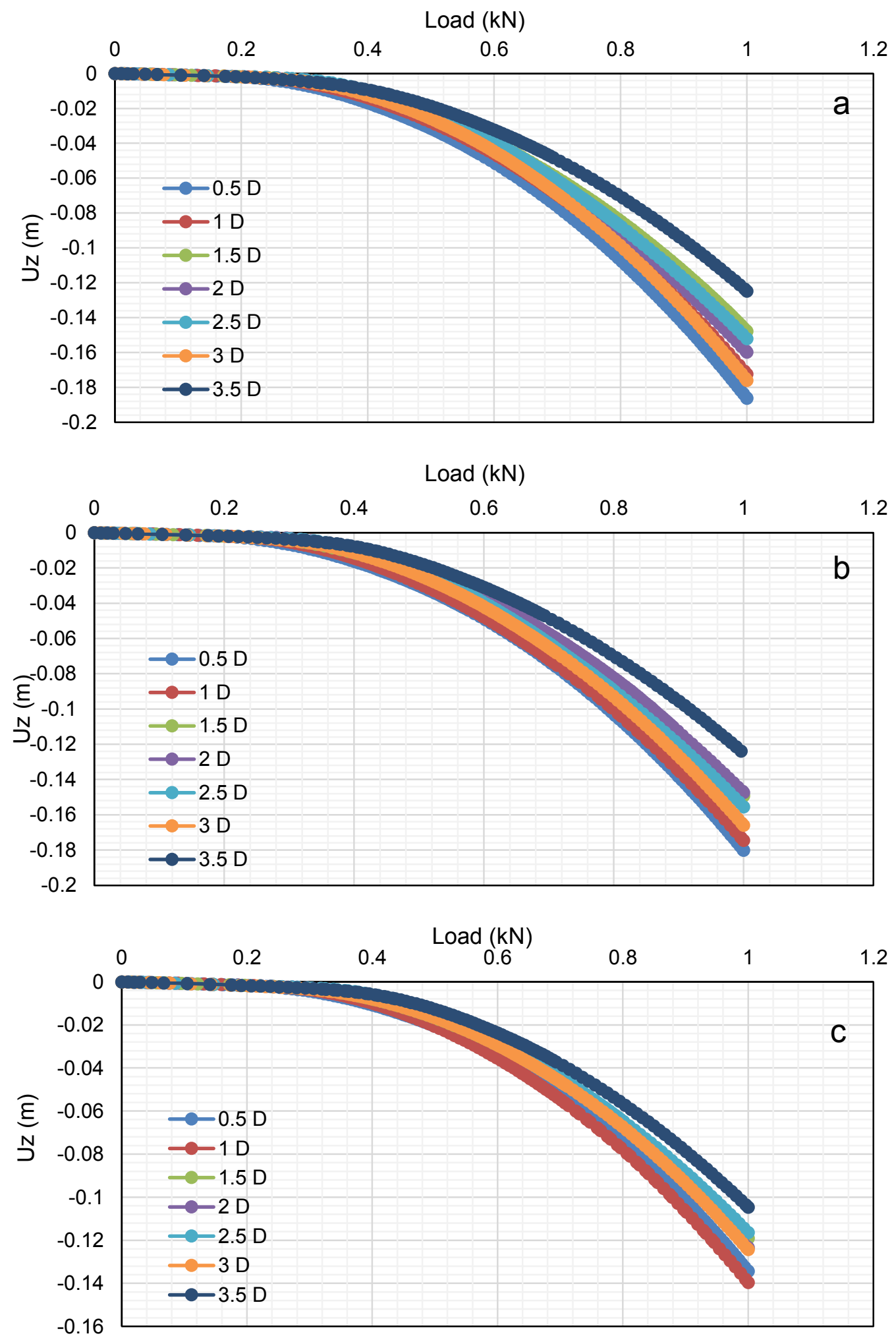

Figure 11. Load-displacement under compression static load with three helices with different spacing for a) top helix, b) middle helix, c) bottom helix.

\section{Conclusions}

This study can be concluded as follows

- The higher the number of helices, the greater the value of the pile capacity compared to the pile without helix. 
- The pile capacity in the case of three helices is more than the pile capacity in the case of two helices.

- The greater the distance between the helix, the greater the value of the pile capacity in the case of two helices and three helices.

- The helical pile with different diameters (tapered) gives the value of pile capacity less than the helical pile with the same diameter of helices. The values of displacement in the case of similar diameter helices are less than in the case of different diameter helices due to the small area of the different diameter helices, which cause an increase in the values of displacement compared to similar diameter helices.

- The higher the helical depth, the lower the displacement value, and the lower the helical depth, the higher the displacement value.

- The highest the helix spacing, the lowest the displacement value (all cases), and the lowest the helix spacing, the highest the displacement value (top and middle).

\section{References}

[1] Kim, D., Baek, K. and Park, K., 2018. Analysis of the bearing capacity of helical pile with hexagonal joints. Materials, 11(10), p.1890.

[2] El-Rahim, A., Hamdy, H.A. and Taha, Y.K., 2013. The compression and uplift bearing capacities of helical piles in cohesionless soil. JES. Journal of Engineering Sciences, 41(6), pp.2055-2064.

[3] Sprince, A. and Pakrastinsh, L., 2009. Helical pile behaviour analysis in different soils. Leonids Pakrastinsh.

[4] Elsherbiny, Z.H. and El Naggar, M.H., 2013. Axial compressive capacity of helical piles from field tests and numerical study. Canadian Geotechnical Journal, 50(12), pp.1191-1203.

[5] Salhi, L., Nait-Rabah, O., Deyrat, C. and Roos, C., 2013. Numerical modeling of single helical pile behavior under compressive loading in sand. EJGE, 18, pp.4319-4338.

[6] Mustafa, K. K., 2018, Experimental analysis of screw pile models with different configurations embedded within cohesionless soil. M.Sc. Thesis, University of Technology, Baghdad, Iraq.

[7] Elkasabgy, M. and El Naggar, M.H., 2015. Axial compressive response of large-capacity helical and driven steel piles in cohesive soil. Canadian Geotechnical Journal, 52(2), pp.224-243.

[8] Sirsikar, R.A., 2018. Study of helical pile behaviour in cohesionless soil. Doctoral dissertation, National Institute of Technology Durgapur.

[9] Ammar, O. K., 2020. Experimental study to evaluate the behavior of partially saturated sandy soils on compression and uplift capacities of helical piles with different helices. M.Sc. Thesis, University of Technology, Baghdad, Iraq.

[10] PLAXIS 3D-Reference Manual (https://communities.bentley.com). 